\title{
Vinculação da gestante e apego materno fetal ${ }^{1}$
}

\author{
Eluisa Bordin Schmidt ${ }^{2}$ \\ Universidade Regional Integrada do Alto Uruguai e das Missões, Erechim-RS, Brasil \\ Irani Iracema de Lima Argimon \\ Pontificia Universidade Católica do Rio Grande do Sul, Porto Alegre-RS, Brasil
}

\begin{abstract}
Resumo: Este trabalho verificou as relações existentes entre o tipo de vinculação da gestante, sintomas de ansiedade, depressão e nível de apego materno fetal. Participaram 136 gestantes entre 18 e 42 anos de idade, do $6^{\circ}$ ao $9^{\circ}$ mês de gestação com escolaridade mínima de $5^{\text {a }}$ série. Foi realizado um estudo quantitativo, transversal, correlacional. Os instrumentos utilizados foram: Ficha de Dados Sociodemográficos, Escala de Vinculação do Adulto (EVA), Escala de Apego Materno Fetal (MFAS), Inventário de Depressão de Beck (BDI) e Inventário de Ansiedade de Beck (BAI). A análise dos dados foi realizada por meio de estatística descritiva, teste exato de Fischer, simulações de Monte Carlo e Correlação de Pearson. Os resultados indicaram que existe associação significativa entre o tipo de vinculação da gestante e o apego materno fetal, e sintomas depressivos. As gestantes que apresentaram uma vinculação segura evidenciaram apego materno fetal alto e sintomas depressivos e ansiosos mínimos.
\end{abstract}

Palavras-chave: gravidez, comportamento de apego, feto, depressão, ansiedade.

\section{Pregnant women's bonding and maternal-fetal attachment}

\begin{abstract}
This study verified the association between the type of pregnant women's bonding, symptoms of anxiety, depression and the level of maternal-fetal attachment. The sample was composed of 136 pregnant women between 18 and 42 years of age, from the sixth to the ninth month of pregnancy, with at least the fifth grade. This is a quantitative, transversal and correlational study. The used instruments were: a social-demographic data form, Adult Bonding Scale (EVA), Maternal-Fetal Attachment Scale (MFAS), the Beck Depression Inventory (BDI), and the Beck Anxiety Inventory (BAI). Data were analyzed through descriptive statistics, Fisher's exact test, Monte Carlo Simulation and Pearson's correlation. Results indicate that there is significant association between the type of pregnant women's bonding, maternal-fetal attachment and depression symptoms. The pregnant women who presented strong bonding showed a high level of maternal-fetal attachment and fewer anxious and depressive symptoms.
\end{abstract}

Keywords: pregnancy, attachment behavior, fetus, major depression, anxiety.

\section{Vínculo de la gestante y apego materno fetal}

\begin{abstract}
Resumen: En este estudio se investigó la relación entre el tipo de vínculo del embarazo, los síntomas de ansiedad y de depresión y el nivel de vínculo materno fetal. Participaron 136 mujeres entre 18 y 42 años de edad, entre los 6 y 9 meses de embarazo y con un mínimo de quinto grado de escolaridad. Se trata de un estudio cuantitativo, transversal, de correlación. Los instrumentos utilizados fueron: Ficha de Datos Sociodemográficos, Escala de Vínculo de Adultos (EVA), Escala de Apego Materno-Fetal (MFAS), el Beck Depression Inventory (BDI) y el Beck Anxiety Inventory (BAI). Los resultados indicaron que existe una significativa asociación entre el tipo de vínculo del embarazo, el apego materno fetal, y los síntomas depresivos. En general, las mujeres embarazadas que tienen un vínculo seguro mostraron un apego materno fetal alto y síntomas depresivos y de ansiedad mínimos.
\end{abstract}

Palabras clave: embarazo, conducta de apego, feto, depresión, ansiedad.

Nos últimos anos, um crescente número de pesquisas (Correia \& Linhares, 2007; Piccinini, Marin, Alvarenga, Lopes, \& Tudge, 2007; Watcher, 2002) têm estudado os processos através dos quais as pessoas estruturam, desenvolvem e mantêm laços afetivos ao longo do ciclo vital. Nessa perspectiva, encontra-se a teoria do apego, igualmente nomeada como teoria da vinculação, formulada por Bowlby (1969/1984). Trata-se de uma concepção teórica do

1 As autoras agradecem à CAPES por viabilizar financeiramente este estudo, à bolsista de Iniciação Científica Marília Schmidt pelo auxílio na formatação do artigo e à assessora estatística Natália Barbieri pelo apoio na formatação das tabelas.

2 Endereço para correspondência:

Prof. Ms. Eluisa Bordin Schmidt. Universidade Regional Integrada do Alto Uruguai e das Missões. Departamento de Ciências Humanas. Curso de Psicologia. Av. Sete de Setembro, 1621. CEP 99.700-000.

Erechim-RS, Brasil.E-mail: eluisabs@via-rs.net desenvolvimento sócio-emocional que considera a existência de uma necessidade humana inata para formar laços afetivos íntimos com pessoas significativas. Essa propensão já estaria presente no neonato em forma embrionária e continuaria na infância, vida adulta e velhice. No período da infância, os vínculos são estabelecidos com os pais ou substitutos que são procurados na busca de conforto, carinho e proteção. $\mathrm{Na}$ adolescência e vida adulta, esses laços persistem, sendo complementados por novos vínculos.

Para explicar tal processo, Bowlby (1989) utilizou o conceito de modelo interno de funcionamento, por meio do qual a criança constrói uma imagem de si mesma e de seus cuidadores que influencia o que ela sente em relação a ambos. Esse modelo desenvolvido durante a infância evolui na medida em que a criança cresce, passando a fazer parte de sua personalidade, transformando-se em uma representação mental da relação de apego, que tende a persistir ao longo da 
vida e exerce influência nas futuras relações afetivas (Bowlby, 1969/1984; Main, Kaplan, \& Cassidy, 1985; Canavarro, 1999; Carvalho, Politano, \& Franco, 2008).

Nos primeiros anos de vida, a reciprocidade dos pais para responder às necessidades da criança e a qualidade da interação favorecem o desenvolvimento de um senso de segurança, uma noção de bem-estar e confiança que será utilizada como base para o conhecimento e exploração do ambiente. A partir dessas experiências, a criança vai estruturando um tipo de apego que é influenciado pela interação que estabelece com seus pais, ou substitutos (Bowlby, 1989; Ainsworth, 1994). Porém, as funções dos laços de vinculação na idade adulta não são idênticas às das crianças nem às dos adolescentes, pois os indivíduos adultos adquiriram uma autonomia que lhes permite prover por si próprios sua sobrevivência (Guedeney \& Guedeney, 2004).

Na perspectiva da estabilidade e continuidade do estilo de apego ao longo do ciclo vital, há uma transmissão intergeracional, processo em que, intencionalmente ou não, atitudes e comportamentos de uma geração precedente influenciam comportamentos numa próxima geração (Lebovici, 1987; Kretchmar \& Jacobitz, 2002; Wachter, 2002; Holz \& Nunes, 2005; Weber, Selig, Bernardi, \& Salvador, 2006).

A qualidade do relacionamento de apego entre os pais e seus progenitores reflete-se em seu estilo de apego com a criança e o modelo internalizado de apego da mãe está relacionado com a habilidade de entender e responder às necessidades de seu filho (Wachter, 2002). Desse modo, a representação mental do adulto, das suas próprias relações precoces de apego, reflete-se nos subsequentes comportamentos parentais (Koren-Karie, 2000).

As investigações sobre apego vêm sendo ampliadas, incluindo o período fetal como um período também de vinculação (Chamberlein, 1994; Piontelli, 1995; Caron, 2002), evidenciando que o apego materno fetal é um preditor do apego pós-natal entre mãe e bebê. Os avanços técnico-científicos têm confirmado a existência de capacidades sensoriais no feto e no bebê recém nascido, e auxiliado a compreensão das interações feto maternas e suas repercussões no plano emocional, tanto da mãe quanto do bebê (Brazelton \& Cramer, 1992; Piontelli, 1995; Klaus \& Klaus, 2001). Ao longo da gravidez, o bebê intra-útero vive experiências e vai sendo influenciado pelas experiências da mãe. Nos últimos meses, o feto já ouve e responde ao toque, dando respostas a estímulos externos, o que possibilita a construção de uma sintonia entre a mãe e o feto (Wilheim, 2000; Busnel, Yasuku, \& Cunha, 2002).

O Apego Materno Fetal (AMF) é um termo usado para descrever os comportamentos e atitudes da mulher de adaptação à gravidez, sendo esses comportamentos baseados em representações cognitivas que incluem o imaginário da mãe, bem como suas atribuições sobre as características físicas e emocionais do feto (Siddiqui \& Hägglof, 2000). Cranley (1981, p. 282) definiu o AMF como "a intensidade com a qual a gestante manifesta comportamentos que representam a afiliação e a integração com sua criança intra-útero".
Vários estudos sintetizados por Doan e Zimerman (2003) investigaram os fatores que poderiam ter correlação com o AMF: (a) características de personalidade da mãe, incluindo empatia, ansiedade e depressão; (b) atitudes para com a gravidez; (c) fatores situacionais vivenciados durante a gestação; (d) apoio social recebido durante o período gestacional; (e) relacionamento marital; (f) características específicas da gravidez, como estágio da gestação, sintomas físicos e planejamento da concepção; (g) fatores demográficos, tais como idade materna e número de gestações; $(\mathrm{h})$ perdas perinatais. Entre as variáveis que positivamente influenciam na intensidade do AMF, está o avanço da idade gestacional, a presença dos movimentos fetais, a história da gravidez e a história de apego da própria mãe (Lerum, Major, \& LoBiondoWood, 1989; Honjo e cols., 2003; Righetti, Dell Avanzo, Grigio, \& Nicolini, 2005), o apoio social dos membros da família e pares (Cranley, 1984; Shin, Park, \& Kim, 2006) e o planejamento da gravidez (Shieh, Kravitz, \& Wang, 2001; Salisbury, Law, LaGasse, \& Lester, 2003).

Considerando o desenvolvimento da criança, o Apego Materno Fetal tem sido conceituado como a primeira parte de um continuum de apego, que começa durante a gravidez e se estende no relacionamento entre a mãe e o bebê no pós-natal (Grace, 1989; Müller \& Ferketich, 1993). Esse conceito foi confirmado pelos estudos e observação de fetos por ultra-sonografia realizados por Piontelli (1995), que reforçam a existência de continuidade de aspectos da vida pré e pós-natal, tendo cada feto características de comportamento que, de alguma forma, continuam na vida pós-natal.

Seguindo na perspectiva da existência de relação entre AMF, apego pós-natal e desenvolvimento da criança, Siddiqui e Hägglof (2000) examinaram a associação entre AMF durante o terceiro trimestre da gravidez e a interação mãebebê nas doze semanas do pós-natal. Os resultados desse estudo indicaram que algumas mães, que experimentaram afeição e fantasiaram mais sobre os seus bebês intra-útero, apresentaram maior envolvimento na interação nas 12 semanas do pós-natal. Outros estudos sobre o apego e desenvolvimento da criança no pós-natal enfatizam que a mãe ocupa papel fundamental na interação e desenvolvimento do bebê (Thomas, Lima, Tavares, \& Oliveira, 2005; Lopes e cols., 2007; Frizzo \& Piccinini, 2007; Piccinini e cols., 2007; Seild-De-Moura e cols., 2008). Sob essa mesma ótica, as pesquisas de Müller e Ferketich (1993) e de Condon e Corkindale (1997) constataram que o apego materno fetal pode ser positivamente correlacionado ao apego mãe-bebê e ao desenvolvimento social, emocional e cognitivo da criança. Para avaliar o AMF, Cranley (1981) desenvolveu a Escala de Apego Materno Fetal (MFSA), que pode ser aplicada durante todo o período gestacional, porém se mostra mais sensível quando aplicada após o $6^{\circ}$ mês de gestação, quando já se evidenciam os movimentos fetais.

Pesquisas sobre as dimensões de apego do adulto e sintomas de ansiedade e depressão mostraram que os modelos de apego podem estar relacionados à manifestação de sintomas depressivos e ansiosos (Hankin, Kassel, \& Abela, 2005, Correia \& 
Linhares, 2007), assim como os sintomas de ansiedade e depressão podem interferir no AMF (Condon \& Corkindale, 1997).

Alguns instrumentos foram estruturados para classificar e avaliar os padrões de apego na idade adulta. A Escala de Vinculação do Adulto (EVA) investiga as relações com os pares, considerando que seu papel é generalizável às relações com outras figuras significativas. Este instrumento indica três padrões de apego: (a) Vinculação Segura: as relações estabelecidas com pares são percebidas como respondendo às necessidades do outro gerando, dessa forma, sensações de segurança e bem-estar; (b) Vinculação Ansiosa: caracterizase pelo desejo de manter os parceiros próximos, existindo hipervigilância a aspectos ligados à separação; a presença e a disponibilidade dos parceiros é percebida como incerta; (c) Vinculação Evitante: caracteriza-se pelas estratégias de diminuição da importância da relação; os pares são percebidos como fontes indutoras de estresse e alvos de desconfiança (Canavarro, 1999).

Tendo em vista que, durante o desenvolvimento, a pessoa estrutura um estilo de apego que se reflete nas suas várias relações afetivas e a relevância que esse padrão de vínculo assume nas interações precoces, tais como a de uma gestante com seu feto e posteriormente com seu filho, o presente estudo buscou verificar as relações existentes entre o tipo de vinculação da gestante e o apego com seu bebê ainda intra-útero e a presença ou não de sintomas de ansiedade e depressão.

\section{Método}

Trata-se de um estudo cujo delineamento é quantitativo, do tipo transversal, correlacional.

\section{Participantes}

Participaram do estudo 136 gestantes primíparas e multíparas, de diferentes níveis socioeconômicos, tendo como critérios de inclusão estarem entre o $6^{\circ}$ e $9^{\circ}$ mês de gestação, ter no mínimo 18 anos de idade, com escolaridade mínima de $5^{\mathrm{a}}$ série, residentes na cidade Erechim-RS.

Após aprovação do projeto de pesquisa pelo Comitê de Ética da Pontifícia Universidade Católica do Rio Grande do Sul, foi realizado contato com as gestantes que foram selecionadas a partir das informações dos prontuários de atendimento ou pelo serviço de enfermagem desses locais, que encaminhavam aquelas que faziam parte dos critérios de inclusão. Após essa triagem, as participantes eram informadas sobre os objetivos da pesquisa, e caso concordassem em participar, assinavam o Termo de Consentimento Livre e Esclarecido.

Foram incluídas as participantes que recebiam atendimento nas Unidades Básicas de Saúde (UBS), faziam parte do programa Materno Infantil da Secretaria de Saúde do Município ou frequentavam grupos de gestantes desenvolvidos em um dos hospitais da cidade no período de outubro a dezembro de 2004 e janeiro de 2005. Foram excluídas da pesquisa grávidas com idade gestacional anterior aos 6 meses, em função dos critérios de aplicação da escala MFSA, com menos de 18 anos e com nível de instrução abaixo da $5^{\text {a }}$ série, de acordo com as normas de aplicação da BAI e BDI, além de questões éticas que requisitariam autorização dos pais para as gestantes com menos de 18 anos. Havia previsão estatística de uma amostra de 90 participantes, como houve boa receptividade dos serviços e a maioria das convidadas se dispôs a participar da pesquisa, foi possível entrevistar 136 grávidas.

\section{Instrumentos}

Para a coleta de dados foram utilizados os seguintes instrumentos: (a) Ficha de Dados Sociodemográficos; (b) Escala de Vinculação do Adulto (EVA); (c) Escala de Apego Materno Fetal (MFAS); (d) Inventário de Depressão de Beck (BDI); (e) Inventário de Ansiedade de Beck (BAI). Os instrumentos são descritos a seguir.

A Ficha de Dados Sociodemográficos corresponde a um questionário criado pelas autoras da pesquisa e consiste de itens referentes à gestante, incluindo idade, estado civil, escolaridade, profissão, nível socioeconômico, idade gestacional, número de filhos, planejamento da gravidez, religião, com quem residia, se contava com outras pessoas para auxiliar no cuidado com o bebê, dados sobre o acompanhamento pré-natal e uso de drogas.

A Escala de Vinculação do Adulto foi construída por Collins e Read (1990) com o objetivo de desenvolver um instrumento estruturado por Hazan e Shaver (1987). Essa escala tem tradução para o português de Portugal e normatização para a mesma amostra (Canavarro, 1999) e identifica três padrões de vinculação: seguro, ansioso e evitante. Composta por dezoito itens, avalia o tipo de vinculação que o indivíduo adulto estabelece com os pares. Para quantificar o tipo de vinculação predominante é utilizada uma pontuação que varia de 1 (nada característico em mim) a 5 (extremamente característico em mim), tendo o item 8 pontuação invertida.

A Escala de Apego Materno Fetal é um instrumento construído por Cranley (1981) para investigar comportamentos que a mulher desenvolve durante a gravidez na preparação para o nascimento de seu bebê. Adaptada e validada para o Brasil por Feijó (1999), contém 24 itens com cinco possibilidades de resposta, que variam de 5 (Quase sempre) a 1 (Nunca), tendo o item 22 pontuação invertida.

O BDI e o BAI, validados para o Brasil por Cunha (2001), são instrumentos para medir a intensidade de sintomas depressivos e de ansiedade respectivamente.

O Inventário de Depressão de Beck é um instrumento utilizado para medida da intensidade de sintomas depressivos. Foi adaptado e validado para o Brasil por Cunha (2000). Trata-se de um inventário de auto-relato de 21 itens, cada um com quatro alternativas, subentendendo graus crescentes de gravidade da depressão com escore de 0 a 3 .

O Inventário de Ansiedade de Beck (BAI) foi construído com base em vários instrumentos de auto-relato, e mede a intensidade de sintomas de ansiedade. Foi adaptado e validado para o Brasil por Cunha (2001) e é constituído por 21 itens, que são afirmações descritivas de sintomas de ansiedade e que devem ser avaliados pelo sujeito com referência 
a si mesmo, em uma escala de 4 pontos que reflete o nível crescente de cada sintoma desde a não apresentação de sintomas até apresentação de sintomas graves. $\mathrm{O}$ escore total é o resultado do escore dos itens individuais e permite a classificação em níveis de intensidade da ansiedade.

\section{Procedimentos}

Foram realizadas reuniões com as direções do hospital e da Secretaria da Saúde do Meio Ambiente do município para explicar os objetivos da pesquisa e obter autorização para contatar as gestantes atendidas nesses serviços. Os instrumentos foram aplicados logo após a realização dos grupos de gestantes, nas salas de espera ou em consultórios disponíveis no local. A coleta de dados foi realizada pela primeira autora e três alunos de iniciação científica do curso de Psicologia da Universidade Regional Integrada, campus de Erechim, previamente treinados pelas pesquisadoras.

\section{Análise de dados}

A análise quantitativa foi efetuada por meio de estatísticas descritivas: médias, desvio padrão, frequências e percentuais foram utilizados para elaboração de um perfil sócio demográfico das participantes. Em todas as análises, o nível de significância utilizado foi 5\%. Utilizou-se estatística inferencial através do teste exato de Fisher ou simulações de Monte Carlo, quando os valores esperados das tabelas eram inferiores a 5, acompanhados de resíduos padronizados, além da Correlação de Pearson.

\section{Resultados}

\section{Características sociodemográficas}

A idade das 136 gestantes que constituíram a amostra variou entre $18(10,3 \%)$ e 42 anos (1,5\%), com média de 27,88 anos. A Tabela 1 apresenta os dados sociodemográficos das 136 gestantes. Como pode ser observado, a maioria das grávidas estava no $8^{\circ}$ mês de gestação. Quanto ao estado civil, $90,4 \%$ tinham companheiros, sendo $52,2 \%$ das gestantes casadas e $38,2 \%$ unidas consensualmente, $89,7 \%$ delas moravam com o pai do bebê, $36,8 \%$ residiam somente com ele e $52,9 \%$ com ele em conjunto com outros familiares e pessoas.

No que se refere à escolaridade, $41,9 \%$ tinham completado o ensino médio, $41,2 \%$ o ensino fundamental e $16,9 \%$ o ensino superior; cerca de metade da amostra era multípara e aproximadamente $53 \%$ delas tinham planejado a gravidez, $66,2 \%$ participaram de grupos de gestantes. No que tange à possibilidade da gestante contar com outras pessoas para auxiliá-la nos cuidados com o bebê após o nascimento, 74,3\% das futuras mães afirmaram que contavam com outras pessoas e com relação ao uso de drogas durante a gestação, 7,4\% das gestantes responderam que usaram de tabaco e álcool.

Entre as participantes do estudo, $85,3 \%$ não tiveram abortos anteriores a esta gestação. Das que tiveram abortos anteriores, $13,2 \%$ foram espontâneos e $1,5 \%$ provocados. A maioria das gestantes $(77,9 \%)$ era católica e $47,1 \%$ não tinha renda própria.
Tabela 1

Dados sociodemográficos de 136 gestantes em atendimento na Unidade Básica de Saúde, no Programa Materno Infantil e no hospital do município

\begin{tabular}{|c|c|c|}
\hline & $n$ & $\%$ \\
\hline \multicolumn{3}{|l|}{ Idade gestacional } \\
\hline 6 meses & 29 & 21,3 \\
\hline 7 meses & 35 & 25,7 \\
\hline 8 meses & 46 & 33,8 \\
\hline 9 meses & 26 & 19,2 \\
\hline \multicolumn{3}{|l|}{ Estado civil } \\
\hline Casada & 71 & 52,2 \\
\hline Solteira & 8 & 5,9 \\
\hline Separada & 5 & 3,7 \\
\hline União Consensual & 52 & 38,2 \\
\hline \multicolumn{3}{|l|}{ Escolaridade } \\
\hline Fundamental & 56 & 41,2 \\
\hline Médio & 57 & 41,9 \\
\hline Superior & 23 & 16,9 \\
\hline \multicolumn{3}{|l|}{ Reside com quem } \\
\hline Só com o pai do bebê & 50 & 36,8 \\
\hline $\begin{array}{l}\text { Pai do bebê e outros } \\
\text { familiares e/ou outras pessoas }\end{array}$ & 72 & 52,9 \\
\hline $\begin{array}{l}\text { Familiares e/ou outras } \\
\text { pessoas sem o pai do bebê }\end{array}$ & 14 & 10,3 \\
\hline \multicolumn{3}{|l|}{ Primíparas } \\
\hline Sim & 66 & 48,5 \\
\hline Não & 70 & 51,5 \\
\hline \multicolumn{3}{|l|}{ Planejamento da gravidez } \\
\hline Sim & 73 & 53,7 \\
\hline Não & 63 & 46,3 \\
\hline \multicolumn{3}{|c|}{ Participação nos grupos de gestantes } \\
\hline $\operatorname{Sim}$ & 90 & 66,2 \\
\hline Não & 18 & 13,2 \\
\hline Não responderam & 28 & 20,6 \\
\hline \multicolumn{3}{|l|}{ Auxílio nos cuidados do bebê } \\
\hline Sim & 101 & 74,3 \\
\hline Não & 35 & 25,7 \\
\hline \multicolumn{3}{|l|}{ Uso de drogas durante a gestação } \\
\hline Não & 126 & 92,6 \\
\hline Fumo & 9 & 6,7 \\
\hline Álcool & 1 & 0,7 \\
\hline \multicolumn{3}{|l|}{ Abortos anteriores } \\
\hline Nenhum aborto & 116 & 85,3 \\
\hline Provocados & 2 & 1,5 \\
\hline Espontâneos & 18 & 13,2 \\
\hline \multicolumn{3}{|l|}{ Religião } \\
\hline Católica & 106 & 77,9 \\
\hline Evangélica & 21 & 15,4 \\
\hline Outros & 4 & 3 \\
\hline Não possui & 5 & 3,7 \\
\hline \multicolumn{3}{|l|}{ Renda da gestante } \\
\hline Não tem renda & 64 & 47,1 \\
\hline Menos de 1 salário & 6 & 4,4 \\
\hline 1 a 3 salários & 48 & 35,3 \\
\hline 4 a 5 salários & 7 & 5,1 \\
\hline Mais de 5 salários & 11 & 8,1 \\
\hline
\end{tabular}


Tipo de vinculação, apego materno fetal, sintomas de depressão e ansiedade

Como pode ser observado na Tabela 2, os resultados obtidos mediante o teste exato de Fisher mostraram que houve associação significativa entre o tipo de vinculação da gestante (seguro, ansioso, evitativo) e o nível de apego materno fetal $(p=0,047)$, indicando que, quando a gestante tinha uma vinculação do tipo seguro, o apego materno fetal foi alto, enquanto na gestante com vinculação evitante e ansiosa o apego materno fetal foi médio.
Da mesma forma, houve associação significativa entre o tipo de vinculação da gestante (seguro, ansioso, evitativo) e sintomas depressivos $(p=0,036)$, pois, entre os três tipos de vinculação, a que apresentou mais sintomas depressivos moderados foi o tipo evitante. Por outro lado, não houve associação significativa entre o tipo de vinculação da gestante (seguro, ansioso, evitativo) e sintomas de ansiedade $(p=0,167)$.

Tabela 2

Relação entre o tipo de vinculação (EVA) de 136 gestantes com niveis de apego materno fetal (MFAS), sintomas depressivos (BDI) e sintomas de ansiedade (BAI)

\begin{tabular}{|c|c|c|c|c|c|c|c|c|c|c|}
\hline \multicolumn{2}{|c|}{ EVA } & \multicolumn{3}{|c|}{ MFAS } & \multicolumn{3}{|c|}{ BDI } & \multicolumn{3}{|c|}{ BAI } \\
\hline & & Médio & Alto & Mínimo & Leve & Moderado & Mínimo & Leve & Moderado & Grave \\
\hline \multirow[t]{2}{*}{ Segura } & $n$ & 46 & 53 & 74 & 18 & 7 & 50 & 30 & 13 & 6 \\
\hline & $\%$ & 46,5 & 53,5 & 74,7 & 18,2 & 07,1 & 50,5 & 30,3 & 13,1 & 06,1 \\
\hline \multirow[t]{2}{*}{ Ansiosa } & $n$ & 04 & 02 & 03 & 03 & 0 & 02 & 03 & 0 & 1 \\
\hline & $\%$ & 66,7 & 33,3 & 50,0 & 50,0 & 0 & 33,1 & 50,0 & 0 & 16,7 \\
\hline \multirow[t]{2}{*}{ Evitante } & $n$ & 22 & 09 & 17 & 07 & 7 & 10 & 09 & 07 & 5 \\
\hline & $\%$ & 71,0 & 29,0 & 54,8 & 22,6 & 22,6 & 32,3 & 29,0 & 22,6 & 16,1 \\
\hline$p$ - valor & & \multicolumn{2}{|c|}{0,047} & \multicolumn{3}{|c|}{0,036} & & \multicolumn{2}{|c|}{0,167} & \\
\hline
\end{tabular}

De acordo com o teste exato de Fisher, houve associação significativa entre com quem a grávida residia e o tipo de vinculação $(p=0,008)$, sendo que as gestantes que residiam somente com o pai do bebê evidenciaram vinculação segura, aquelas que residiam com o pai do bebê junto com outros familiares apresentaram vinculação evitante e aquelas que residiam somente com familiares e/ou outras pessoas sem o pai do bebê demonstraram vinculação ansiosa.

\section{Nível de AMF, sintomas depressivos e de ansiedade e variáveis sóciodemográficas}

Por meio do teste exato de Fisher, não se encontrou associação significativa entre AMF e sintomas depressivos $(p=0,449)$ e sintomas de ansiedade $(p=0,493)$.

Como pode ser visto na Tabela 3 houve associação entre:

(1) Escolaridade e $A M F(p=0,034)$ : as gestantes com ensino fundamental, incompleto, apresentaram menores valores de AMF, enquanto as que tinham escolaridade maior (superior) apresentaram nível de AMF mais alto;

(2) Com quem a gestante reside e o nivel de $A M F$ $(p=0,045)$ : quando a gestante residia com familiares e/ou outras pessoas tendia a ter um AMF mais baixo, enquanto aquela que residia somente com o pai do bebê apresentou um AMF mais alto;

(3) Primeira gravidez e AMF $(p=0,010)$ : comparativamente a primípara tinha um AMF significativamente mais alto que as mulheres multíparas;

(4) Uso de drogas durante a gestação e $A M F(p=0,036)$ : as gestantes que usavam drogas (álcool e tabaco) durante a gestação apresentaram um AMF mais baixo quando comparadas àquelas que não usavam essas drogas durante a gestação.

Como pode ser visto também na Tabela 3, o teste exato de Fisher indicou que não houve associação significativa entre o AMF em: aborto anterior espontâneo $(p=0,333)$, se contava com alguém para auxiliar nos cuidados com o bebê $(p=0,238)$, uso de drogas antes da gestação $(p=0,352)$, estado civil $(p=0,356)$, religião $(p=0,190)$, renda $(p=0,560)$, planejamento da gravidez $(p=0,231)$, participação em grupo de gestantes $(p=1,000)$. De acordo com o teste qui-quadrado, não se encontrou associação entre a idade gestacional e AMF $(p=0,053)$. Por meio da correlação de Pearson, não houve associação significativa entre a idade materna e $\operatorname{AMF}(p=0,428$ e $r=0,068)$.

\section{Sintomas de ansiedade, depressão, aborto anterior e idade gestacional}

$\mathrm{Na}$ Tabela 4 , no que se refere à presença de sintomas de ansiedade, de acordo com o teste exato de Fisher, estes se apresentaram associados à presença de aborto anterior provocado $(p=0,008)$, em que as mulheres que tiveram esse tipo de aborto tendiam a ter nível de ansiedade grave. Por outro lado, não se encontrou evidência de haver associação significativa entre aborto e sintomas depressivos $(p=0,864)$. Também não foi encontrada evidência de haver associação significativa entre a idade gestacional e sintomas de ansiedade $(p=0,901)$ e entre idade gestacional e sintomas depressivos $(p=0,199)$. 
Tabela 3

Relação entre os dados sociodemográficos de 136 gestantes e o nível de apego materno fetal (MFAS)

\begin{tabular}{|c|c|c|c|c|c|c|c|}
\hline \multirow[b]{2}{*}{ Dados sociodemográficos } & \multicolumn{3}{|c|}{ MFAS } & \multirow[b]{2}{*}{ Dados sociodemográficos } & \multicolumn{3}{|c|}{ MFAS } \\
\hline & $\begin{array}{c}n \\
(\mathbf{\%})\end{array}$ & Médio & Alto & & $\begin{array}{c}n \\
(\%)\end{array}$ & Médio & Alto \\
\hline Escolaridade & & & & Drogas antes da gestação & & & \\
\hline Ensino Fundamental Incompleto & & $26(70,3)$ & $11(29,7)$ & Nenhuma & & $57(50,4)$ & $56(49,6)$ \\
\hline Ensino Fundamental Completo & & $9(47,4)$ & $10(52,6)$ & Fumo & & $13(68,4)$ & $6(31,6)$ \\
\hline Ensino Médio & & $31(54,3)$ & $26(45,6)$ & Álcool & & $1(33,3)$ & $2(66,7)$ \\
\hline $\begin{array}{l}\text { Superior } \\
p \text { - valor }\end{array}$ & \multicolumn{3}{|c|}{0,0034} & Mais do que um dos anteriores & & $1(100)$ & $0(0)$ \\
\hline Reside com quem & & & & $p$ - valor & & \multicolumn{2}{|c|}{0,352} \\
\hline Pai do bebê (somente) & & $21(42)$ & $29(58)$ & Estado civil & & & \\
\hline $\begin{array}{l}\text { Pai do bebê e/ou outros familiares/ } \\
\text { pessoas }\end{array}$ & & $40(55,6)$ & $32(44,4)$ & Casada & & $35(49,3)$ & $36(50,7)$ \\
\hline & & & & Solteira & & $6(75)$ & $2(25)$ \\
\hline do bebê & & $11(78,6)$ & $3(21,4)$ & Separada & & $4(80)$ & $1(20)$ \\
\hline$p$ - valor & \multicolumn{3}{|c|}{0,045} & União Consensual & & $27(51,9)$ & $25(48,1)$ \\
\hline Primeira gravidez & & & & $p$ - valor & & \multicolumn{2}{|c|}{0,356} \\
\hline Sim & & $27(40,9)$ & $39(59,1)$ & Religião & & & \\
\hline Não & & $45(64,3)$ & $25(35,7)$ & Não possui religião & & $1(20)$ & $4(80)$ \\
\hline $\begin{array}{l}p \text { - valor } \\
\text { Drostacão }\end{array}$ & \multicolumn{3}{|c|}{0,01} & Católica & & $57(53,8)$ & $49(46,2)$ \\
\hline $\begin{array}{l}\text { Drogas durante a gestaçao } \\
\text { Nenhuma }\end{array}$ & & $63(50)$ & $63(50)$ & Luterana & & $2(100)$ & $0(0)$ \\
\hline Fumo & & $8(88,9)$ & $1(11,1)$ & Evangélica & & $12(57,1)$ & $9(42,9)$ \\
\hline Álcool & & $1(100)$ & $0(0)$ & Espírita & & $0(0)$ & $2(100)$ \\
\hline$p$ - valor & \multicolumn{3}{|c|}{0,036} & $p$ - valor & & \multicolumn{2}{|c|}{0,19} \\
\hline Aborto anterior espontâneo & & & & Renda Mensal & & & \\
\hline $\operatorname{Sim}$ & & $13(65)$ & $7(35)$ & Não tem renda & & $37(57,8)$ & $27(42,2)$ \\
\hline Não & \multirow{2}{*}{\multicolumn{3}{|c|}{0,333}} & Menos de 1 salário & & $2(33,3)$ & $4(66,7)$ \\
\hline $\begin{array}{l}p \text { - valor } \\
\text { Auxílio nos cuidados do bebê }\end{array}$ & & & & 1 a 3 salários & & $26(54,2)$ & $22(45,8)$ \\
\hline Sim & & $50(49,5)$ & $51(50,5)$ & 4 a 5 salários & & $3(42,9)$ & $4(57,1)$ \\
\hline Não & & $22(62,9)$ & $13(37,1)$ & Mais de 5 salários & & $4(36,4)$ & $7(63,6)$ \\
\hline$p$ - valor & \multicolumn{3}{|c|}{0,238} & $p$ - valor & & \multicolumn{2}{|c|}{0,56} \\
\hline
\end{tabular}

Tabela 4

Relação entre sintomas de ansiedade e depressão, aborto anterior e idade gestacional

\begin{tabular}{|c|c|c|c|c|c|c|c|c|c|}
\hline & & \multicolumn{4}{|c|}{ BAI } & \multicolumn{3}{|c|}{ BDI } & \multirow[b]{2}{*}{ Total } \\
\hline & & Mínimo & Leve & Moderado & Grave & Mínimo & Leve & Moderado & \\
\hline \multicolumn{10}{|c|}{ Aborto Anterior } \\
\hline \multirow[t]{2}{*}{ Nenhum } & $n$ & 52 & 40 & 15 & 9 & 81 & 23 & 12 & 116 \\
\hline & $\%$ & 44,8 & 34,5 & 12,9 & 7,8 & 69,8 & 19,8 & 10,3 & 100 \\
\hline \multirow[t]{2}{*}{ Espontâneo } & $n$ & 10 & 2 & 5 & 1 & 13 & 4 & 1 & 18 \\
\hline & $\%$ & 55,6 & 11,1 & 27,8 & 5,6 & 72,2 & 22,2 & 5,6 & 100 \\
\hline \multirow[t]{2}{*}{ Provocado } & $n$ & 0 & 0 & 0 & 2 & 0 & 1 & 1 & 2 \\
\hline & $\%$ & 0 & 0 & 0 & 100 & 0 & 50 & 50 & 100 \\
\hline \multirow{2}{*}{ Total } & $n$ & 62 & 42 & 20 & 12 & 94 & 28 & 14 & 136 \\
\hline & $\%$ & 45,6 & 30,9 & 14,7 & 8,8 & 69,1 & 20,6 & 10,3 & 100 \\
\hline$p$ - valor & & & & & & & 0,864 & & \\
\hline \multicolumn{10}{|c|}{ Idade gestacional } \\
\hline \multirow[t]{2}{*}{6 meses } & $n$ & 14 & 10 & 3 & 2 & 25 & 2 & 2 & 29 \\
\hline & $\%$ & 48,3 & 34,5 & 10,3 & 6,9 & 86,2 & 6,9 & 6,9 & 100 \\
\hline \multirow[t]{2}{*}{7 meses } & $n$ & 14 & 11 & 7 & 3 & 22 & 7 & 6 & 35 \\
\hline & $\%$ & 40 & 31,4 & 20 & 8,6 & 62,9 & 20 & 17,1 & 100 \\
\hline \multirow[t]{2}{*}{8 meses } & $n$ & 21 & 14 & 8 & 3 & 29 & 12 & 5 & 46 \\
\hline & $\%$ & 45,7 & 30,4 & 17,4 & 6,5 & 63 & 26,1 & 10,9 & 100 \\
\hline \multirow[t]{2}{*}{9 meses } & $n$ & 13 & 7 & 2 & 4 & 18 & 7 & 1 & 26 \\
\hline & $\%$ & 50 & 26,9 & 7,7 & 15,4 & 69,2 & 26,9 & 3,8 & 100 \\
\hline \multirow{2}{*}{ Total } & $n$ & 62 & 42 & 20 & 12 & 94 & 28 & 14 & 136 \\
\hline & $\%$ & 45,6 & 30,9 & 14,7 & 8,8 & 69,1 & 20,6 & 10,3 & 100 \\
\hline$p$ - valor & & & \multicolumn{2}{|c|}{0,901} & & & 0,199 & & \\
\hline
\end{tabular}




\section{Discussão}

A teoria do apego e os estudos posteriores indicam que a idade adulta é caracterizada pela estabilidade dos comportamentos de vinculação, que se baseiam nos modelos internos de funcionamento (Waters, Hamilton, \& Weinfield, 2000; Stein e cols., 2002). No adulto, os estilos de apego são reatualizados nas relações de vinculação, por meio da relação amorosa e do acesso ao papel de progenitor com seus próprios filhos (Guedeney \& Guedeney, 2004).

A partir dos resultados deste estudo, que buscou verificar a associação entre o tipo de vinculação da gestante e o AMF, pode-se pensar que o tipo de vinculação da gestante pode ser um preditor de AMF, uma vez que as gestantes com vinculação segura evidenciaram nível de AMF alto. Neste sentido, Mikulincer e Florian (1999) examinaram o estilo de apego da mulher e AMF e encontraram que mulheres com vinculação segura são fortemente apegadas aos seus fetos, enquanto mulheres com estilo de apego evitativo denotaram fraco apego pré-natal. De certa forma, elas continuavam a funcionar como faziam com suas primeiras figuras de vinculação, recriando o mesmo padrão de trocas que tiveram na sua infância, o que, segundo Kretchmar e Jacobitz (2002), Fonagy (1999) e Weber e cols. (2006) pode estar na base de um fenômeno de transmissão intergeracional e influenciar as práticas educativas e os relacionamentos parentais.

De acordo com a teoria do apego, fatores contextuais estressantes ativam o sistema de apego internalizado, o que leva a respostas comportamentais e emocionais. No caso das gestantes, a transição para a maternidade torna-se um fator de estresse que ativa o modelo interno de funcionamento de apego, em consequência, a manifestação de sintomas depressivos e ansiosos poderá ocorrer, entre outros fatores, pelo tipo de apego internalizado. Nesta pesquisa, que também investigou o tipo de vinculação da gestante e os sintomas de ansiedade e depressão, evidenciou-se associação significativa entre o estilo de vinculação da gestante e sintomas depressivos. Quando foi analisada a presença de sintomas depressivos moderados e comparada à diferença entre os tipos de vinculação, observou-se que as gestantes com apego evitativo apresentaram frequência maior de sintomas depressivos em nível moderado.

Confirmando esses achados, estudos prévios demonstraram que as mulheres com estilo de apego ansioso ou evitativo eram particularmente mais vulneráveis a apresentarem sintomas depressivos e depressão (Lidgren, 2001). O apego evitativo também se mostrou associado a baixos níveis de afetividade positiva em mulheres com altos níveis de sintomas depressivos (Adam, Gunnar, \& Tanaka, 2004). Na transição para a parentalidade, mulheres com tipo de apego ansioso e evitante experimentaram sintomas elevados de depressão em estágio posterior ao nascimento dos bebês, seis meses após o parto (Simpson, Rholes, Campbell, Tran, \& Wilson, 2003).

Um dado significativo foi que a maioria das gestantes que demonstrou um tipo de vinculação segura residia somente com o pai do bebê e tinha AMF alto, enquanto as gestantes que apresentavam padrão de vinculação evitante moravam com o pai do bebê e outros familiares e apresentavam AMF médio. Da mesma forma, as gestantes que residiam com os familiares e/ou outras pessoas sem o pai do bebê, foram as que apresentaram apego ansioso e AMF médio.

Em investigações sobre o tipo de apego internalizado e como este interage nas percepções da mulher em relação à sustentação recebida pelo companheiro, um estudo de Simpson e cols. (2003) demonstrou que as mulheres com apego ansioso percebiam o apoio do esposo como deficiente, tanto na gravidez, como no pós-parto, e apresentavam um aumento de sintomas depressivos nestes períodos. Considerando que gravidez é um período de mudanças, com novas exigências biopsicossociais, esse resultado também aponta para a importância da presença do companheiro neste período. Piccinini, Lopes, Gomes e De Nardi (2008) ressaltam que na gravidez ocorrem mudanças corporais, psicológicas e interpessoais que influenciam a dinâmica individual e as demais relações sociais da gestante, que vivenciam intensos sentimentos em relação ao tornar-se mãe. Hernandes e Hutz (2008) reforçam que para as mulheres o ajustamento conjugal e emocional é importante principalmente no período da gravidez do primeiro filho. Sendo assim, uma das fontes de apoio social para o desenvolvimento do apego está na capacidade de adaptação do casal à nova experiência. Huth-Bocks, Levendosky, Bogat e Van Eye (2004) ampliam essas questões para o período pós-natal, afirmando que o apoio social está relacionado a maior capacidade de cuidados maternos e à presença de apego seguro do bebê no primeiro ano de vida. Rapoport e Piccinini (2006), em revisão de estudo teóricos e empíricos que relacionavam apoio social e maternidade, indicaram que, especialmente em situações estressantes, o apoio social aumenta a responsividade materna beneficiando tanto a relação mãe bebê quanto conjugal.

Não se encontrou associação significativa entre AMF e sintomas depressivos e de ansiedade na amostra estudada. Buscou-se então, analisar se esse resultado estaria ligado à idade gestacional. Quando analisada a presença de sintomas depressivos e de ansiedade, e correlacionada à idade gestacional, considerando os três últimos meses (período investigado no estudo), não houve associação significativa. Considera-se o achado como positivo, pois os baixos níveis de ansiedade e depressão em relação ao AMF podem ter gerado como resultado geral da amostra um bom nível de apego. Esse resultado é compatível com estudos de revisão, como o de Alhusen (2008) que avaliando as pesquisas publicadas entre 2000 e 2007, refere que fatores como depressão, abuso de substâncias e alto nível de ansiedade estão associados a um baixo nível de AMF. Esse achado também é confirmado pelos estudos de Rodrigues, Pérez-López e Brito de la Nuez (2004) e Hart e McMahon (2006) que indicam que menos sintomas de ansiedade estão associados a um nível mais alto de vinculação pré-natal.

Embora todas as gestantes tenham apresentado bom nível de $\mathrm{AMF}$, as primíparas revelaram um AMF mais alto quando 
comparadas às mulheres que já tinham outros filhos. Esse dado é confirmado pela literatura, que aponta que as diferenças entre primíparas e multíparas estão ligadas a uma maior expectativa das primeiras em relação ao nascimento do bebê (Mercer \& Ferketich, 1994; Nichols, Roux, \& Harris, 2007). Do mesmo modo, o grau de escolaridade da gestante influenciou de forma positiva o AMF, sugerindo que o fator educacional pode ser importante para a promoção do apego entre a mãe e seu feto.

Observou-se que as mulheres que usaram tabaco e álcool durante a gestação manifestaram um AMF mais baixo. Esse dado sugere que a gestante que faz uso de substância química apresenta menos interação com seu bebê intra-útero, o que já foi observado em estudos anteriores (Alhusen, 2008).

A maioria das variáveis sociodemográficas não se mostrou relacionada ao nível de AMF. Cranley (1981) aborda que o apego de uma mulher ao seu feto dependeria basicamente de comportamentos e tarefas (tais como os investigados pela MFAS) que a mulher realiza no desenvolvimento da gravidez, e não de fatores demográficos.

A literatura indica que as variáveis sociodemográficas que influenciam o AMF seriam o avanço da idade gestacional, os movimentos fetais, o apoio social dos membros da família e dos pares (Doan \& Zimerman, 2003). Tais dados foram confirmados nesta pesquisa, pois, de modo geral, o nível de AMF foi médio e alto. Este resultado pode ser atribuído também, ao fato das gestantes entrevistadas já estarem no último trimestre da gravidez, período em que os movimentos fetais já estavam instaurados, o que pode ter influenciado nos resultados positivos em relação aos níveis de apego.

Os achados desse estudo confirmam que há relação entre o padrão de vinculação da gestante e o nível de AMF, enfatizando que as gestantes que apresentaram vinculação segura evidenciaram AMF alto e sintomas depressivos e ansiosos mínimos.

\section{Considerações finais}

Este estudo demonstrou que a gravidez é uma etapa do ciclo vital em que já começam a se estruturar as relações de apego, o modelo interno de funcionamento da gestante está associado à intensidade de apego que ela estabelece com seu bebê no período fetal. Contudo, como aborda Guedeney e Guedeney (2004), os estilos de apego não são estruturados rigidamente, podendo mudar de acordo com os acontecimentos e as experiências significativas que gerem mudança no comportamento de qualquer dos indivíduos que fazem parte da relação de apego.

Embora os resultados desta pesquisa não tenham indicado associação significativa entre o AMF e participação de grupo de gestantes, essas receberam acompanhamento do serviço de saúde e participaram de várias atividades educativas, o que pode sugerir que, quando acompanhada, a grávida pode evidenciar níveis de apego mais altos, auxiliando no fortalecimento do apego entre ela e seu bebê, consideração corroborada por estudo de Piccinini e cols. (2008).
A avaliação do nível de ansiedade e depressão materna é relevante para identificação de riscos na saúde mental da gestante e no desenvolvimento do bebê (Correia \& Linhares, 2007; Frizzo \& Piccinini, 2007). Nesse sentido, pode-se destacar que intervenções precoces, como acompanhamento às gestantes em que possam ser identificados aspectos vulneráveis, tais como: altos níveis de sintomas de ansiedade e depressão, baixo nível educacional, se não residem com o pai do bebê e se usam drogas durante a gestação, tornam-se muito importantes.

Dessa forma, essas gestantes podem ser prioritariamente acompanhadas pelos serviços de saúde, que podem funcionar como apoio àquelas mais vulneráveis, contribuindo no campo da prevenção precoce e na estruturação de laços emocionais positivos que terão repercussão ao longo da vida.

\section{Referências}

Adam, E. K., Gunnar, M. R., \& Tanaka, A. (2004). Adult attachment, parent emotion, and observed parenting behavior: Mediator and moderator models. Child Development, 75, 110-122.

Ainsworth, M. (1994). Attachements and other affectional bonds across the life cycle. In C. M. Parkes, J. StevensonHinde, \& F. Marris (Eds.), Attachement across the life cycle. London: Tavistock/Routledge.

Alhusen, J. L. (2008) A literature update on maternal-fetal attachment. Journal Obstetric Gynecology Neonatal Nursing, 37, 315-28.

Bowlby, J. (1984). Apego: A natureza do vínculo. São Paulo: Martins Fontes. (Original publicado em1969)

Bowlby, J. (1989). Uma base segura: Aplicações clínicas da teoria do apego. Porto Alegre: Artes Médicas.

Brazelton, T. B., \& Cramer, N. G. (1992). As primeiras relações. São Paulo: Martins Fontes.

Busnel, M. C.,Yasaku, S., \& Cunha, I. (2002). Relação mãefeto: Visão atual das neurociências. São Paulo: Casa do Psicólogo.

Canavarro, M. C. (1999). Relações afetivas e saúde mental. Coimbra, Portugal: Quarteto.

Caron, N. A. (2002). O ambiente intra-uterino e a relação materno-fetal. In N. A. Caron (Org.), A relação pais-bebê: Da observação à clínica. São Paulo: Casa do Psicólogo.

Carvalho A. M. A., Politano I., \& Franco, A. L. S. (2008). Vínculo interpessoal: Uma reflexão sobre diversidade e universalidade do conceito na teorização na psicologia. Estudos de Psicologia (Campinas), 25, 233-240.

Cranley, M. S. (1981). Development of a tool for the measurement of maternal attachment during pregnancy. Nursing Research, 30, 282-284.

Cranley, M. S. (1984). Social support as a factor in the development of parents attachment to their unborn. Birth Defects: Original Articles Series, 20, 99-124. 
Chamberlein, D. B. (1994). How pre and perinatal psychology can transform the word. Pre and Perinatal Psychology Journal, 8, 187-189.

Collins, N., \& Read, S. (1990). Adult attachment style, working models, and relationship quality in dating couples. Journal of Personality and Social Psychology, 58, 644-663.

Condon, J. T., \& Corkindale, C. (1997). The correlates of antenatal attachment in pregnant women. British Journal Medical Psychology, 70, 372- 379.

Correia, L. L., \& Linhares, M. B. M., (2007). Ansiedade materna nos períodos pré e pós-natal: Revisão da literatura. Revista Latino-Americana de Enfermagem, 15, 677-683.

Cunha, J. A. (2001). Manual da versão em português das Escalas Beck. São Paulo: Casa do Psicólogo.

Doan, H., \& Zimerman, A. (2003). Conceptualizing prenatal attachment: Toward a multidimensional view. Journal of Prenatal and Perinatal Psychology and Health, 18, 109-129.

Feijó, M. C. (1999). Validação brasileira da Maternal-Fetal Attachment Scale. Arquivos Brasileiros de Psicologia, 51(4), 52-62.

Frizzo G. B., \& Piccinini, C. A. (2007). Depressão materna e a interação triádica pai-mãe-bebê. Psicologia: Reflexão $e$ Crítica, 20, 351-360.

Fonagy, P. (1999). Persistencias transgeneracionales del apego: Una nueva teoría. Revista de Psicoanálisis [Versión electrónica]. Recuperado em 05 julho 2004, de http:// www.aperturas.org/3 fonagy.html

Grace, J. (1989). Development of maternal-fetal attachment during pregnancy. Nursing Research, 38, 228-232.

Guedeney, N., \& Guedeney, A. (2004). Vinculação: Conceitos e aplicações. Lisboa: Climpesi.

Hankin, B. L., Kassel, J. D., \& Abela, J. R. Z. (2005). Adult attachment dimensions and specificity of emotional distress symptoms: Prospective investigations of cognitive risk and interpersonal stress generation as mediating mechanisms. Personality and Social Psychology Bulletin, 31, 136-151.

Hart R., \& McMahon C. A. (2006). Mood state and psychological adjustment to pregnancy. Archives Womens Mental Health, 9, 329-37.

Hazan, C., \& Shaver, P. (1987). Romantic love conceptualized as an attachment process. Journal of Personality and Social Psychology, 52, 511-524.

Hernandes J. A. E., \& Hutz C. S. (2008). Gravidez do primeiro filho: Papéis sexuais, ajustamento conjugal e emocional. Psicologia: Teoria e Pesquisa, 24, 133-141.

Holz, A., \& Nunes, M. L. (2005). Vínculos afetivos maternos e comportamento de seus filhos. In S. Areosa \& M. L. Nunes (Orgs.), Psicologia da criança e desenvolvimento humano. Santa Cruz do Sul, RS: EDUNISC.
Honjo, S., Arai, S., Kaneko, H., Ujiie, T., Murase, S., Sechiyama, H., Sasaki, Y., Hatagaki, C., Inagaki, E., Usui, M., Miwa, K., Ishiara, M., Hashimoto, O., Nomura, K., Itakura, A., \& Inoko, K. (2003). Antenatal depression and maternal-fetal attachment. Psychopathology, 36, 304-311.

Huth-Bocks, A., Levendosky, A., Bogat, G., \& Van Eye, A. (2004). The impact of maternal characteristics and contextual variables on infant-mother attachment. Child Development, 75, 480-496.

Koren-Karie, N. (2000). Attachment representations in adulthood: Relations with parental behaviors. The Israel Journal of Psychiatry and Related Sciences, 37, 178-189.

Klaus, M., \& Klaus, P. (2001). Seu surpreendente recémnascido. Porto Alegre: Artmed.

Kretchmar, M. D., \& Jacobitz, D. B. (2002). Observing mother-child relationships across generations: Boundary patterns, attachment, and the transmission of care giving. Family Process, 41, 351-374.

Lebovici, S. (1987). O bebê, a mãe e o psicanalista. Porto Alegre: Artes Médicas.

Lerum, C. W., Major, R. N., \& Lobiondo-Wood, G. (1989). The relationship of maternal age, quickening, and physical symptoms of pregnancy to the development of maternal-fetal attachment. Birth, 16, 13-17.

Lopes, R. C. S., Oliveira, D. S., Vivian, A. G., Bohmgahren, L. M. C., Piccinini, C. A., \& Tudge, J. (2007). Sentimentos maternos frente ao desenvolvimento da criança aos doze meses: Convivendo com as aquisições infantis. Psicologia: Teoria e Pesquisa, 23, 5-15.

Lindgren, K. (2001). Relationships among maternal-fetal attachment, prenatal depression, and health practices in pregnancy. Research in Nursing \& Health, 24, 203-207.

Main, M., Kaplan, N., \& Cassidy, J. (1985). Security in infancy, childhood, and adulthood: A move to the level of representation. Monographs of the Society for Research in Child Development, 50, 66-104.

Mercer, R., \& Ferketich, S. (1994). Predictors of maternal role competence by risk status. Nursing Research, 43, 38-43.

Mikulincer, M., \& Florian, V. (1999). Maternal fetal boding, coping strategies, and mental health during pregnancy: The contribution of attachment style. Journal of Social and Clinical Psychology, 18, 2555-2276.

Müller, M., \& Ferketich, S. (1993). Factor analysis of the Maternal Fetal Attachment Scale. Nursing Research, 42, 144-147.

Nichols, M. R., Roux, G. M., \& Harris, N. R. (2007). Primigravid and multigravid women: Prenatal perspectives. Journal Perinatal Educacion, 16(2), 21-32.

Piccinini, C. A., Lopes, R. S., Gomes, A. G., \& De Nardi, T. (2008). Gestação e a constituição da maternidade. Psicologia em Estudo, 13, 63-72. 
Piccinini, C. A., Marin, A. H., Alvarenga, P., Lopes, R. C. S., \& Tudge, G. R. (2007). Responsividade materna em famílias de mães solteiras e famílias nucleares no terceiro mês de vida da criança. Estudos de Psicologia (Natal), 12, 109-117.

Piontelli, A. (1995). De feto a criança: Um estudo observacional e psicanalitico. Rio de Janeiro: Imago.

Rapoport, A., \& Piccinini, C. A. (2006). Apoio social e experiência da maternidade. Revista Brasileira de Crescimento e Desenvolvimento Humano, 16(1), 85-96.

Righetti, P. L., Dell'Avanzo, M., Grigio, M., \& Nicolini, U. (2005). Maternal/paternal attachment and fourthdimensional ultrasound technique: A preliminary report. British Journal of Psychology, 96, 129-137.

Rodrigues, A. R., Pérez-López, J., \& Brito de la Nuez, A. G. (2004). La vinculación afectiva prenatal y la ansiedad durante los últimos tres meses del embarazo en las madres y padres tempranos: Un estudio preliminar. Anales de Psicología, 20, 93-102.

Salisbury, A., Law, K., Lagasse, L., \& Lester, B. (2003). Maternal fetal attachment. JAMA, 289, 1701.

Seild-De-Moura, M. L., Ribas, A. F. P., Seabra, K. C., Pessôa, L. F., Nogueira, S. E., Mendes, D. M. L. F., Rocha, S. B., \& Vicente, C. C. (2008). Interações mãe-bebê de um e cinco meses: Aspectos afetivos, complexidade e sistemas parentais predominantes. Psicologia: Reflexão e Crítica, 21, 66-73.

Shieh, C., Kravitz, M., \& Wang, H. (2001). What do we know about maternal-fetal attachment? The Kaohsiung Journal of Medicinal Sciences, 17, 448-454.

Shin, H., Park, Y. J., \& Kim, M. J. (2006). Predictors of maternal sensitivity during the early postpartum period. Journal of Advanced Nursing, 55, 425-34.

Siddiqui, A., \& Hägglof, B. (2000). Does maternal prenatal attachment predict postnatal mother-infant interaction? Early Human Development, 59, 13-25.

Simpson, J., Rholes, W. S., Campbell, L., Tran, S., \& Wilson, C. L. (2003). Adult attachment, the transition to parenthood, and depressive symptoms. Journal of Personality and Social Psychology, 84, 1172-1187.

Stein, H., Koontz, A., Fonagy, P., Allen, J., Fultz, J., Brethour, J., Allen, D., \& Evans, R. (2002). Adult attachment: What are the underlying dimensions? Psychology and Psychotherapy: Theory, Research and Practice, 75, 77-91.

Thomas, A. C. P., Lima, M. R. T., Tavares, C. H. F., \& Oliveira, C. G. (2005). Relações afetivas entre mães e recém-nascidos a termo e pré-termo: Variáveis sociais e perinatais. Estudos de Psicologia (Natal), 10, 139-146.

Watcher, M. P. K. (2002). Psychologycal distress and dyadic satisfaction as predictors of maternal fetal attachment. Dissertation Illinois Institute of Technology, 63, 2080.
Waters, E., Hamilton, C. E., \& Weinfield, N. S. (2000). The stability of attachment from infancy to adolescence and early adulthood: General introduction. Child Development, 71, 678-683.

Weber, L. N. D., Selig, G. A., Bernardi, M. G., \& Salvador, A. P. V. (2006). Continuidade dos estilos parentais através das gerações: Transmissão intergeracional de estilos parentais. Paideia (Ribeirão Preto), 16, 407-414.

Wilheim, J. (2000). Psiquismo pré e perinatal. In N. A. Caron (Org.), A relação pais-bebê: Da observação à clínica. São Paulo: Casa do Psicólogo.

Eluisa Bordin Schmidt é Professora Titular do curso de Psicologia da Universidade Regional Integrada do Alto Uruguai e das Missões, campus de Erechim.

Irani Iracema de Lima Argimon é Professora Adjunta da Faculdade de Psicologia da Pontifícia Universidade Católica do Rio Grande do Sul.
Recebido: $14 / 05 / 2008$

$1^{a}$ revisão: $27 / 02 / 2009$

$2^{a}$ revisão: $30 / 03 / 2009$

$3^{a}$ revisão: $28 / 04 / 2009$

Aceite final: $30 / 04 / 2009$ 